www.jmscr.igmpublication.org

Impact Factor 5.84

Index Copernicus Value: 83.27

ISSN (e)-2347-176x ISSN (p) 2455-0450

crossref DOI: _https://dx.doi.org/10.18535/jmscr/v5i8.50

\title{
Bilateral Renal Metastasis with Renal Vein Thrombosis Due to Squamous Cell Carcinoma Esophagus; A Rare Case and A Brief Review of Literature
}

\author{
Authors \\ Simran Kaur ${ }^{1}$, Vikas Makkar ${ }^{2}$, Harmandeep Singh Chahal ${ }^{3}$, Suman Sethi ${ }^{4}$, \\ Amit Dhiman 5 , J.S. Sandhu' \\ ${ }^{1,4}$ Assistant Professor, Department of Nephrology, ${ }^{2,6}$ Professor, Department of Nephrology \\ ${ }^{3}$ Assistant Professor, Department of Urology, ${ }^{5}$ Associate Professor, Department of Oncology \\ 1,2,3,4,5,6 Dayanand Medical College and Hospital, Ludhiana, Punjab, India
}

\section{ABSTRACT}

Kidney is a rare site for metastatic deposits. Out of the solid organ malignant neoplasms, lung cancer is the most common source of renal mets. ${ }^{1}$ Esophageal carcinoma constitutes only $5 \%$ of such deposits. ${ }^{2}$ The renal involvement due to squamous cell carcinoma esophagusis mostly bilateral, with some case reports of solitary deposits, often diagnosed on autopsy, but renal vein thrombosis due to such metastasis has not been reported till date. We report a rare case of a 70 year old male, treated for squamous cell carcinoma esophagus, presenting with bilateral metastasis in kidneys with bilateral renal vein thrombosis.

KEY WORDS: Squamous cell carcinoma esophagus, renal metastasis, renal vein thrombosis.

\section{BACKGROUND}

Metastases from a primary tumor may mimic renal tumors. This is especially the case when kidneys are the only known metastatic site and the primary tumor is treated. The primary tumors commonly metastasizing to kidney are lung, breast, head and neck, prostate and colon, besides unusual sites such as adrenal gland, skin, tibia, testis and esophagus. There have considerable advances in the diagnosis, surgical techniques and chemo-radiotherapy for esophageal carcinoma, but it still remains one of the most lethal cancers and most patients die from its recurrence or metastasis, with a five-year survival rate ranging from $15 \%$ to $25 \%$. $^{3}$ Kidney ranks fifth among the sites harboring deposits from metastasizing esophageal cancer. ${ }^{4}$ The renal involvement is usually bilateral, with occasional case presenting assolitarysol, but renal vein thrombosis due to esophageal metastatic deposits has not been reported till date.

\section{METHOD}

The present case is of a70 years male, type- 2 diabetic of 4 years duration with baseline serum creatinine $1.6 \mathrm{mg} / \mathrm{dl}$, who was diagnosed to have moderately differentiated squamous cell carcinoma of middle one-third of the esophagus (T:3 N:1 M:0) eighteen months back while evaluation for dysphagia. $\mathrm{He}$ had regional lymphnodal metastasis with no distant metastasis on baseline PET scan and received chemotherapy for 3 months followed by surgery (Transthoracic total esophagectomy +Mediastinal lymphdenectomy + Esophago-gastrectomy, with formation of stomach tube). After surgery he remained asymptomatic and on regular follow-up for 15 months, with no evidence of residual disease or 
recurrence. He presented to us with history of dullpain in left lumbar region and left iliac fossa, constipation, loss of appetite and increasing generalized weakness over the past one month. There was no history of radiation of abdominal pain, fever, burning micturition, decreased urine output or hematuria. History of NSAID intake for pain was present. On investigations he had low hemoglobin $(6.8 \mathrm{gm} / \mathrm{dL})$ with normal total and differential count. The renal function test showed blood urea- $48 \mathrm{mg} / \mathrm{dl}$ and serum creatinine -2.37 $\mathrm{mg} / \mathrm{dl}$. First urine report showed protein $1+$, with no RBC or pus cells. Upper GI endoscopy was normal with anastomotic site healthy. Ultrasound showed bulky kidneys with increased echogenicity, normal corticomedullary differentiation and no hydronephrosis. CECT abdomen showed markedly enlarged left kidney

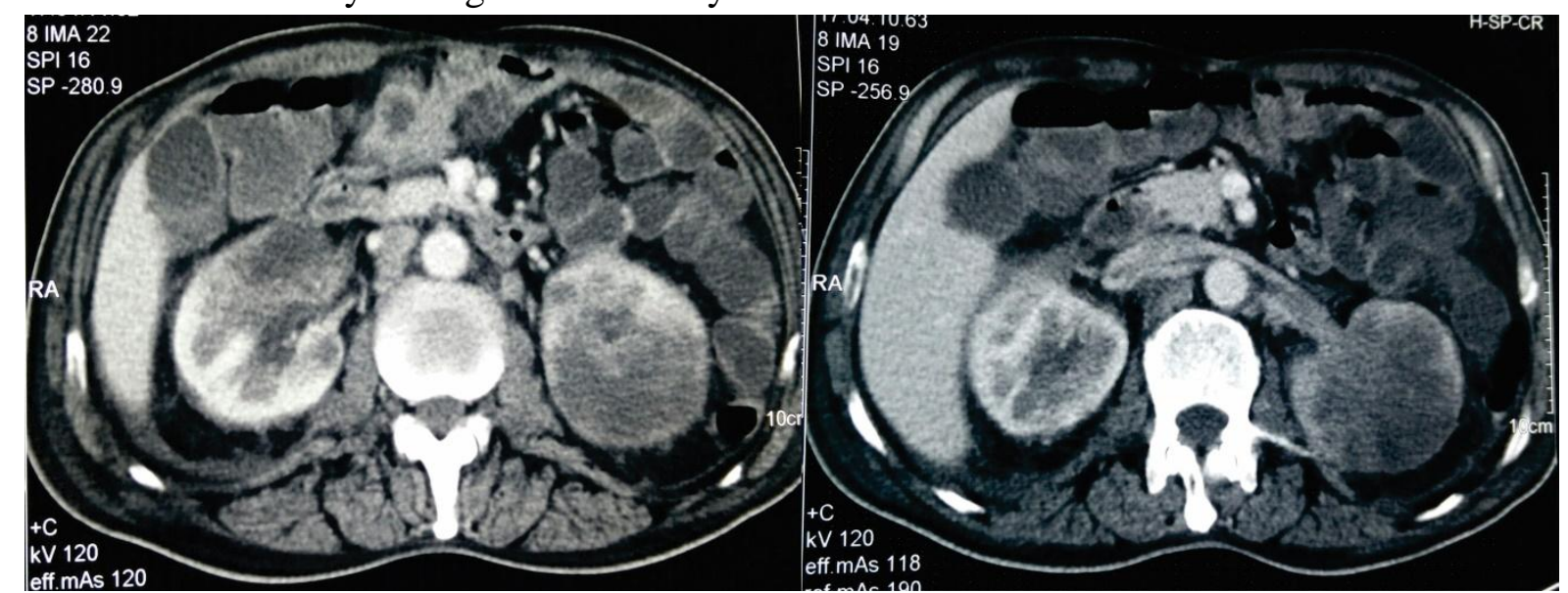

Figure 1: CECT showing ill- defined hypodense lesion diffusely infiltrating the kidney (Left >Right) with infiltration of the left pelvicalyceal system (fig 1a, 1b) and filling defect suggestive of thrombosis in the left renal vein (fig. $1 b$ )
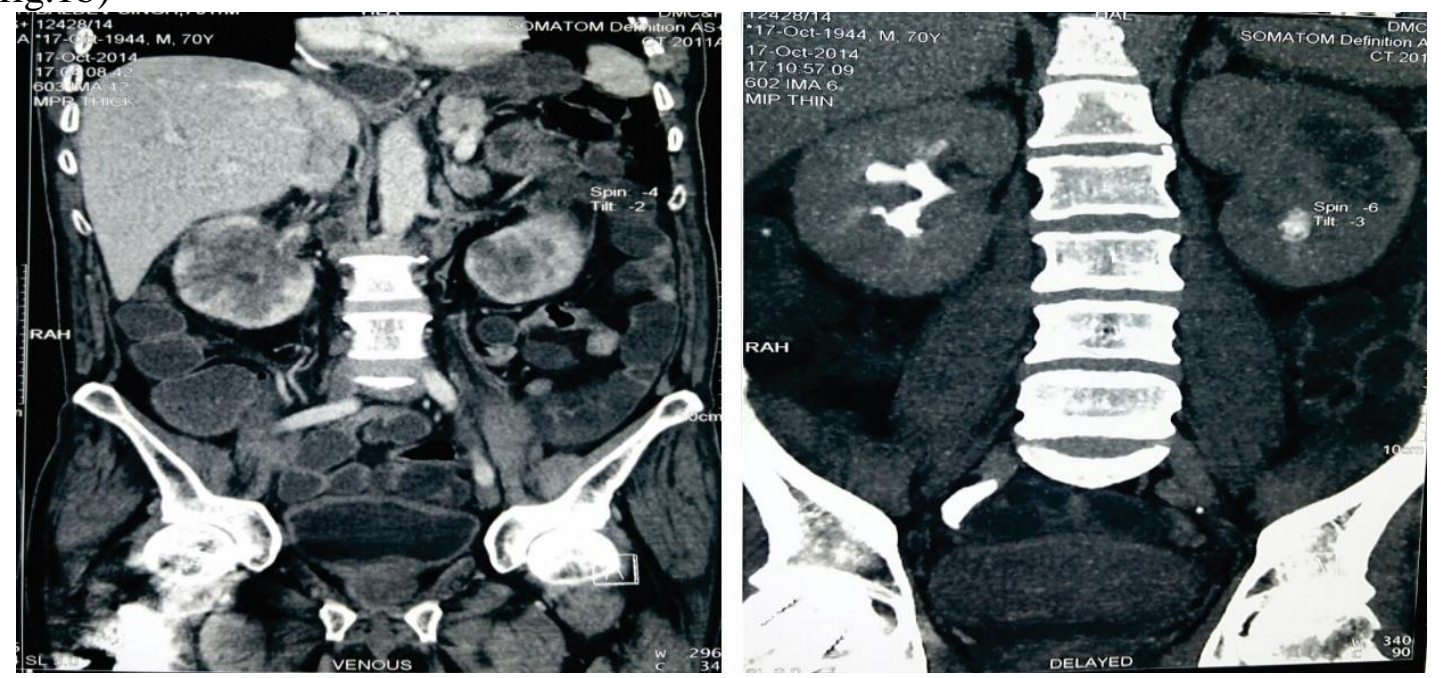

Figure 2: CECT showing a filling defect suggestive of thrombosis in the right renal vein (fig.2a). Left kidney showing obliteration of calyces by the metastatic deposists with excretion of contrast only in the spared calyces (fig. 2b) 
Ultrasound guided biopsy from the renal masson right side showed infiltration of renal parenchyma by clusters of large pleomorphic hyperchromatic cells having well defined cell margin with immunohistochemistry positive for cytokeratin (CK5/6), consistent with metastatic squamous cell carcinoma.

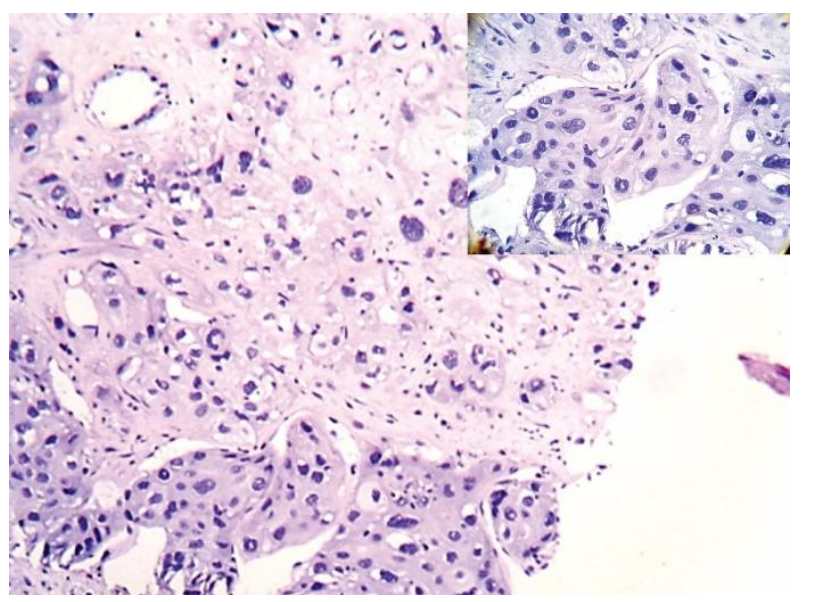

Figure 3: Photomicrograph showing infiltration of the renal parenchyma by clusters of large pleomorphic hyperchromatic cells having well defined cell margin.

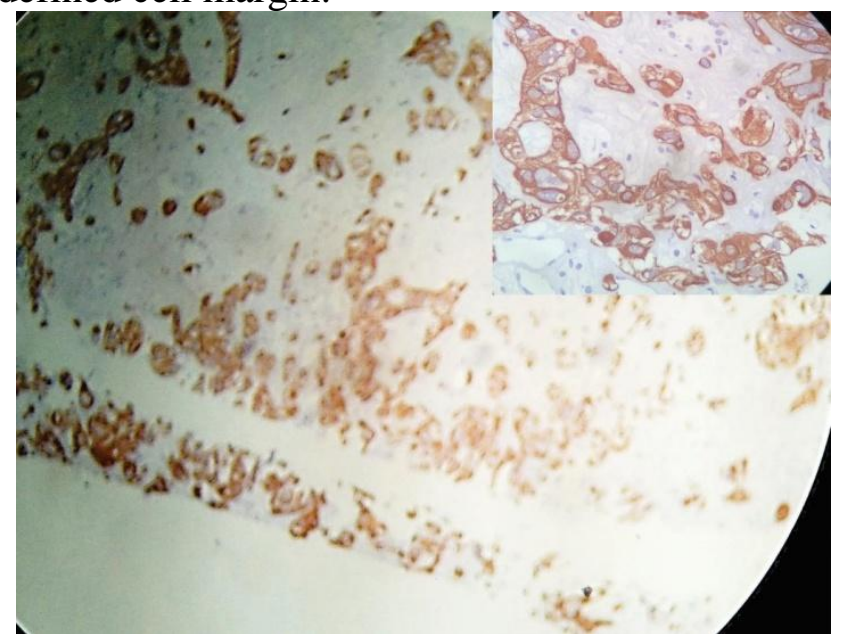

Figure 4: Immunohistochemistry positive for Cytokeratin (CK5/6).

Subsequent renal doppler showed right renal artery having slightly high resistance flow at intraparenchymal level with RI of 0.77 - secondary to raised parenchymal resistance with no visualized venous flow and left renal artery showing patchy flow with no flow inleft renal vein, suggesting bilateral renal vein thrombosis. Patient unfortunately had rapid progression of renal failure and became oliguric within next 15 days and was initiated on hemodialysis (HD). He was non-compliant with dialysis and fluid restriction and died after 3 months while on the way to hospital after missed HD sessions.

\section{DISCUSSION}

Worldwide, oesophageal cancer is the eighth most common cancer and ranks among the top six causes of mortality due to cancers. ${ }^{3}$ Although 50\% to $70 \%$ patients can be treated and cured by surgery, half of them suffer from local recurrences or metastasis even after complete resection. ${ }^{5}$ The most common sites of metastasis are the liver, lung, bone and adrenal glands. ${ }^{6}$ Metastases of esophageal carcinoma in the kidney are considered to be extremely rare. ${ }^{4}$ Squamous cell carcinoma has the highest occurrence among metastatic renal tumors from esophageal carcinoma. Their diagnoses can be synchronous or asynchronous with respect to the primary. Metastases from a primary tumor treated previously may mimic renal tumors. Most renal metastases are multifocal with widespread non renal metastases. Hematuria and proteinuria are seen in less than $20 \%$ of these patients. ${ }^{7}$ Our patienttoo had previously treated squamous cell carcinoma esophagus, with bilateral renal masses, presenting asynchronously to the primary tumor. Most of these patients are asymptomatic, despite extensive renal involvement. This leads to clinical latency, often leading to diagnosis on autopsy, attributed to ignorance of such rare metastases while the patient is alive. ${ }^{8}$ This patient had subtle abdominal symptoms probably due to progressing renal vein thrombosis. We did not come across any case in the literature reporting renal vein thrombosis due to metastatic disease. Because of the rarity of renal metastasis of esophageal carcinoma, no conclusive treatment has been established yet according to the European Association of Urology (EAU) guidelines and National Comprehensive Cancer Network (NCCN) guidelines. The median survival following the detection of a recurrent or metastatic esophageal carcinoma is two to ten months. ${ }^{7}$ This patient had rapidly progression to end stage renal disease which we hypothesized to be due to progressive renal metastasis with bilateral renal 
vein thrombosis possibly compounded by radio contrast nephropathy. Patient survived for three months on palliative therapy and hemodialysis.

\section{CONCLUSION}

In summary, we describe a rare case of bilateral renal metastasis with bilateral renal vein thrombosis from squamous cell carcinoma esophagus presenting eighteen months after treatment. The suspicion of renal metastasis from a previously treated primary carcinoma should be high on cards whenever patient presents with subtle non-specific complaints.

\section{CONSENT}

Written informed consent was obtained from the patient's son for the publication of this report and accompanying images.

\section{COMPETING INTERESTS}

Authors declare that there are no conflicts of interest.

\section{REFERENCES}

1. Bracken RB, Chica G, Johnson DE, Luna M. Secondary renal neoplasms: an autopsy study. South Med J 1979; 72: 806-7.

2. Grise P, Botto H, Camey M. J Urol.Esophageal cancer metastatic to kidney: report of 2 cases. 1987 Feb; 137(2): 274-6.

3. Gupta B, Kumar N. Worldwide incidence, mortality and time trends for cancer of the oesophagus. Eur J Cancer Prev. 2016 [doi: 10.1097/CEJ.0000000000000249] [Epub ahead of print].

4. Lai YC, Lai JI, Lin PC, Wang WS. Esophageal Squamous Cell carcinoma with Solitary Renal Metastasis-A Case Report. Journal of Gastroenterology and Hepatology Research 2012; 1(9): 226-28.

5. Mariette C, Balon JM, Piessen G, Fabre S, Van Seuningen I, Triboulet JP. Pattern of recurrence following complete resection of esophageal carcinoma and factors predictive of recurrent disease. Cancer 2003; 97: 1616-23.

6. Quint LE, Hepburn LM, Francis IR, Whyte RI, Orringer MB. Incidence and distribution of distant metastases from newly diagnosed esophageal carcinoma. Cancer 1995; 76: 1120-5.

7. Sun Y, Yu X, Zhang Y. Renal metastasis after esophagectomy of esophageal squamous cell carcinoma: a case report and literature review. World Journal of Surgical Oncology 2014; 12:165.

8. Chang KP, Huang CP, Chang H. Solitary renal metastasis of esophageal squamous cell carcinoma mimicking primary renal neoplasm - A case report and literature review. Biomedicine (Taipei). 2016 Mar; $6(1): 6$. 